\title{
COMMINUTION EMPLOYING LIQUID NITROGEN PRETREATMENTS
}

by

\author{
S. C. Yen \\ Department of Civil Engineering and Mechanics \\ E. J. Hippo \\ Department of Mechanical Engineering and Energy Processes \\ Southern Illinois University at Carbondale \\ Carbondale, lllinois 62901
}

Final Report

January 1, 1989-August 31, 1990

\author{
Submitted to \\ U.S. Department of Energy \\ Contract Number DE-FC22-89PC89904
}

November 1990 


\section{DISCLAIMER}

This report was prepared as an account of work sponsored by the United States Government. Neither the United States nor any agency thereof, nor any of their employees, makes any warranty, express or implied, or assumes any legal liability or responsibility for the accuracy, completeness, or usefulness of any information, apparatus, product, or process disclosed, or represents that its use would not infringe privately owned rights. Reference herein to any specific commercial product, process, or service by trade name, mark, manufacturer, or otherwise, does not necessarily constitute or imply its endorsement, recommendation, or favoring by the United States Government or any agency thereof. The views and opinions of authors expressed herein do not necessarily state or reflect those of the United States Government or any agency thereof. 


\begin{abstract}
This report presents a summary of the work conducted during the past year. This includes the study of fracture development in coal, freezing temperature enhanced grinding, indentation fracture, pyrite/minerals separation, and envisioned coal cleaning systems. In the fracture study, we found that cracks develop along the interface of pyrite (or minerals) and coal under a freezing temperature environment. The interfacial cracks indicate that the liberation of pyrite can be very easy. The density of fracture in coal was the greatest under the liquid nitrogen environment. A smaller number of cracks was found in the dry ice and dry-iced acetone treated coal. With a longer duration of freezing temperature treatment, the interfacial microcracks expand into the coal matrix. Such an expansion of cracks further enhanced the grindability of coal. In other words, the lower the pretreated temperature, the greater the size reduction that can be obtained. The dry ice pretreatment did improve the size reduction over that of the untreated coal. It appears that a desired particle size distribution of ground coal can be controlled by varying the time and temperature of the freezing temperature pretreatment process. The indentation fracture study indicated that damage developed as a result of the freezing. temperature treatments. Such damage resulted in the loss of the load transferring mechanism of coal. It was found that the fracture toughness of coal decreases with a decrease in the pretreatment temperature. In the pyrite liberation study, we found that more than $90 \%$ of pyrite was freed from the coal matrix after grinding for coal treated at the freezing temperature below 155K. In particular, for liquid nitrogen treated coal, approximately $95 \%$ of pyrite was liberated. Such a high pyrite liberation has never been reported in the literature. In the study of the envisioned process, a fluidized bed coal cleaning system was designed. The concept of this fluidized bed system is based on the findings obtained in this project. This fluidized bed coal cleaning system will allow a selective liberation mechanism, freezing temperature pretreatments (at a wide range of temperature), coal grinding, recirculation of warm and cold gases, etc. A freezing temperature environmental chamber and an impactor have been designed and built to study the feasibility of the fluidized bed process. We are optirnistic about the operation capability of this system. A very conservative initial economic analysis of the fluidized bed system revealed that approximately $\$ 8$ to $\$ 10$ in operation cost is required to clean a ton of coal.
\end{abstract}




\section{SUMMARY}

Desulfurization and demineralization of Illinois coal have been less effective than desired because of the distribution of pyrite and other minerals as microparticles in the coal. Efforts at effective cleaning have been directed toward finely grinding the coal to release these unwanted particles. However, another approach to breaking the physical bond between the coal and unwanted minerals is possible.

Coal treated to low temperature quenching to create cleavage of the physical bond between coal and mineral particles (especially pyrite) has shown enhanced separation during grinding without the need for fine grinding (Yen and Hippo 1989a,b). This reduces the grinding, physical cleaning, and handling costs associated with producing low levels of pyrite and other mineral matter.

The freezing temperature pretreatment suggests that pyrite and minerals may be easily liberated from the coal matrix. This is because interfacial cracks between pyrite and coal develop as a result of the freezing temperature treatment. Pretreatment using both dry ice and dry-iced acetone can only produce a small amount of interfacial crack. Significant pyrite/coal interfacial cracks were generated only with the freezing temperature pretreatment at a temperature below 190K. The dry ice pretreatment gave no improvement of size reduction over the untreated coal. The dry-iced acetone pretreatment gave a slight improvement in size reduction. Based on this, the study of the effect of liquid nitrogen pretreatment on coal rleaning was emphasized.

Reduction of fracture resistance was found for the coal sample being treated with freezing temperatures. For the liquid nitrogen treated coal a $50 \%$ reduction of the fracture resistance was found.

If a longer duration of freezing temperature treatment is used ultrafine coal particles can be obtained using a mild grinding process. This is due to the fact that 
interfacial cracks have expanded into the coal matrix. As a result, the fracture resistance of coal is greatly decreased. Grinding of treated coal also produces ground coal with a very narrow band of particle size distribution. For the liquid nitrogen pretreated coal, the medium particle size is $20 \mu \mathrm{m}$. Crinding efficiency was found to vary with different grinders. In general, a centrifugal ball mill gave a greater size reduction over other types of mills used in this project. It was found that the higher the grinding speed the greater size reduction.

One of the goals of this project is to demonstrate the effectiveness of low temperature pretreatment of coal as an enhancement for pyrite/minerals liberation through grinding. The pyrite liberation evaluation was conducted using an AIA system. It was found that pyrite liberation increased with a decrease in the freezing temperature level. For the untreated coal, approximately $70 \%$ of pyrite was liberated. For the coal treated below $155 \mathrm{~K}$, more than $90 \%$ of pyrite was liberated. For the liquid nitrogen treated coal, as much as $95 \%$ of pyrite was liberated. It is believed that pyrite liberation using freezing temperature pretreatment is a feasible concept.

Based on the results obtained in the grinding study and the pyrite liberation evaluation, it is believed that the amount of pyrite liberation can be predicted for different freezing temperature pretreatment and grinding conditions. In addition, the particle size distribution of ground coal (pyrite, coal, etc.) can also be controlled. Such a feature may become important when selective coal grinding and pyrite liberation are desirable for a coal cleaning process.

In the envisioned process study, a fluidized bed based coal cleaning system is proposed and is currently being analyzed and designed. The concept of this fluidized coal cleaning system is based primarily on the findings of this research. The importance of this fluidized bed system is that it can be used to study a wider fraezing temperature range. The major componenis of this system include a fluid bed with grinding mechanism, piping system, compressor, heat exchanger, and 
selective separation mechanisms. A freezing temperature chamber and an impactor were designed and built to study the feasibility of this system. Based on our initial calculations, this fluidized bed may prove to be economical when considered as a full-scale coal cleaning plant. The exact economic analysis of this cleaning system cannot be performed unless well-defined operating parameters (i.e, treatment temperature, grinding conditions, etc.) have been identified and defined. However, it is estimated that an operating cost of approximately $\$ 8$ to $\$ 10$ is required to clean a ton of coal for a 20-year lifetime fluidized bed coal cleaning system. Further, more detailed economic analysis will be performed when the optimized operating condiiions of the fluidized bed system is determined.

\section{INTRODUCTION AND BACKGROUND}

Coal desulfurization procedures are concerned with removing both pyritic sulfur and sulfur organically bound to the coal matrix. Coal cleaning technologies include water cleaning, gravity separation, and froth flotation methods. While these methods are relatively low in cost, they can be used to remove only approximately 30 to $50 \%$ of the pyritic sulfur from coal (U.S. Department of Energy 1987). This degree of desulfurization will not meet the current environmental standards established by the EPA. Based on recent evaluations conducted by PETC, three generic coal cleaning technologies (selective agglomeration, heavy-liquid cycloning, and advanced flotation) are thought to be capable of removing 90 to $95 \%$ of the pyritic sulfur and also recovering 90 to $95 \%$ of the Btu content (U.S. Department of Energy 1987). On the other hand, the desired level of success has not been achieved to date. Since the particle size of the minerals in coal can be as fine as submicron in diameter (Hamblem et al. 1980, Grady 1977), the complete liberation of pyritic minerals, especially at microcrystalline sizes, requires ultrafine grinding of coal (Bose 1983). Unfortunately, current grinding processes are not considered satisfactory for 
producing ultrafine coals. The grinding of coal is a difficult and complicated process. At the beginning of a grinding process, the coal is brittle due to its micro- and macroflaws. Later, during the grinding process, the coal heats up and becomes ductile (Klimpel 1987). As a result, the coal becomes more and more difficult to grind. It has been found that the duration and energy required for an ultrafine coal grinding process increases exponentially with fine coal particles produced (Smith and Odekirk 1985, Kowalczeinski et al. 1984).

Coal grinding is a complex process which includes stress application, energy consumption comminution, changes in size distribution, flow of ground coal and mineral particles, particle separation, fluid flow, drying, and wear of machines. The grinding process for preparation of pulverized fuel and for liberation of pyrite from coal has been industrialized for several decades (Austin et al. 1984). Traditional grinding technologies have been designed to grind minerals that are brittle. Since coal is a viscoelastic glass, these processes may not be as applicable to the comininution of coal. Coal shows different hardness in its constituent organic components (Hsieh 1976) and also exhibits a certain degree of plasticity (Klimpel 1987) which has been seen in the formation of aggregates during comminution (Parish 1967). As a result, coal behaves as a ductile material and is not easily ground.

Pyrite liberation in coal as a key to sulfur reduction during beneficiation is well established (McCarthey et al. 1969). The liberation mechanism is related to the particle size distribution of pyrite and associated minerals. This is particularly true when a coal sample is pulverized and washed by the float-sink method (Richardson and Love 1979). It has been reported that the structure of coal plays a significant role in size reduction. The profusion of micro- and macro-flaws has been reported as the main reason for the ease of coal grinding at the earlier stage of the process (Klimpel 1987). Therefore, for a more effective coal grinding process, it is necessary to increase the 
brittleness of coal. It is believed that coal, like most other materials, will become more brittle in low temperature environments (Hartwig 1978).

Coal treated to low temperature to create cleavage of the bond between coal and mineral particles (especially pyrite) has shown enhanced separation during grinding without the need for fine grinding (Yen and Hippo 1989a,b). This reduces grinding, physical cleaning and handling costs associated with producing low levels of pyrite and other mineral matter.

The goal of this project is to develop a methodology that will lead to the establishment of an effective, efficient technique for ultrafine grinding of coal. To be more specific, we believe that the key to successful coal grinding is strongly dependent upon the change of the brittleness of coal under a freezing temperature pretreatment. Furthermore, a cryogenic grinding process may provide the basis for the development of advanced technologies involving the separation of the pyritic minerals from coal. These advanced techniques may include the selective liberation of finely dispersed minerals without the need for ultrafine micronization of the coal. The specific objectives of the program are to:

1. determine the effect of low temperature pretreatments on the microfracture development along the coal/pyrite interface and on the fracture resistance (brittleness) of coal. Specifically, we intend to examine the effect of direct contact of coal with liquid nitrogen, dry ice, and dry-iced acetone. Also, we intend to study pyrite liberation as a result of these treatments. This will lead to identification of the minimum temperature for effective low temperature enhanced grinding.

2. determine the fracture resistance of coal under different low temperature pretreatments; 
3. determine the relationships between the fracture resistance of coal and the effectiveness of a grinding process;

4. determine the effect of the froken coal grinding on the pyrite liberation;

5. evaluate factors which might effect process design, scale-up, and economics; and

6. make a first pass economic assessment of the process.

\section{EXPERIMENTAL PROCEDURES}

Coal samples at different pyritic sulfur contents were used as the samples for this research (WYODAK; Pittsburgh; and IBC-101, an Illinois No. 6). The experimental conditions investigated included the time duration of low temperature treatments, time of curing, methods of grinding, and duration of grinding. Typically, a sample first received an initial analysis to determine its sulfur/mineral content and to investigate its coal/mineral interfaces. Then, a portion of the sample was used to determine the indentation fracture toughness. The remaining portion of the sample was subjected to a given low temperature treatment, followed by an AlA inspection for possible fracture along the pyrite/coal interface resulting from the treatment. To determine the effect of low temperature treatment on the fracture resistance of coal, a portion of the pretreated sample received the indentation fracture testing. The remainder of the pretreated coal was then subjected to a given grinding process. After the grinding, the coal powder was inspected through AIA for the pyrite liberation evaluation. Through the pyrite liberation evaluation process, the total percent pyrite/minerals liberation, the range of different particle sizes, and the particle size distribution due to a given low temperature pretreatment can be determined. Brief descriptions of the experiments used in this project are given below.

Low temperature treatments. Initially, liquid nitrogen, dry ice and dry-iced acetone were used to pretreat coal samples. Later, a cooling chamber was built to 
include the study of the freezing temperature effect at various temperature levels between 300 and 77K. Different durations of low temperature soaking were used to pre-condition the coal samples, including samples which did not receive the low temperature treatment. The overall test matrix of the freezing temperature pretreatments is given in Table 1. After the low temperature treatment, some

Table 1

Test Matrix of Freezing Temperature Pretreatment

\begin{tabular}{|c|c|c|c|c|c|c|c|c|c|c|}
\hline \multirow{2}{*}{$\begin{array}{c}\text { Duration } \\
(\min )\end{array}$} & \multicolumn{10}{|c|}{ Freezing Temp̈eratures (K) } \\
\hline & 300 & 250 & 190 & 175 & 155 & 130 & 110 & 77 & Dry Ice & $\begin{array}{l}\text { Dry-Iced } \\
\text { Acetone }\end{array}$ \\
\hline 2 & & & & & & & & $x$ & $x$ & $x$ \\
\hline $\begin{array}{r}5 \\
10\end{array}$ & & $\begin{array}{l}x \\
x\end{array}$ & $\begin{array}{l}x \\
x\end{array}$ & $\begin{array}{l}x \\
x\end{array}$ & $\begin{array}{l}x \\
y\end{array}$ & $\begin{array}{l}x \\
x\end{array}$ & $\begin{array}{l}x \\
x\end{array}$ & $x$ & $x$ & $\begin{array}{l}x \\
x\end{array}$ \\
\hline 15 & & $x$ & & $x$ & & & & $\begin{array}{l}x \\
x\end{array}$ & $\begin{array}{l}x \\
x\end{array}$ & $x$ \\
\hline 20 & & & & & & & & $x$ & $x$ & $x$ \\
\hline 40 & & & & & & & & $x$ & $x$ & $x$ \\
\hline
\end{tabular}

samples immediately undergo the grinding process, and remaining samples are stored and cured at different time intervals in a controlled environment (which will protect the coal powder from oxidation) under the ambient temperature. It is believed that the storage process may allow additional time for the stress-relieving effect and/or fracture to occur. It should be pointed out that, after the liquid nitrogen treatment, a portion of each sample was used to conduct the indentation fracture testing.

Coal grinding. To perform the coal grinding, a low speed ball mill, an attrition mill, a small-scale centrifugal ball mill and an impactor were used. This allowed us to assess the effect of forcing mechanism and speed on the grinding of coal. Different grinding durations were used. All of the grinding mills were used to grind the 
samples treated with liquid nitrogen, dry ice, and dry-iced acetone (Table 2). For the samples treated in the freezing temperature chamber, only two grinding times ( 5 and $10 \mathrm{~min}$ ) using the centrifugal ball mill were used (Table 3). This allowed study of the effect of the duration of grinding on the particle size distribution and pyrite liberation. To investigate the effect of the grinding speed on the pyrite liberation process, the centrifugal ball mill was operated at different rpms (ranging from 100 to 640). After the coal grinding process, the coal powder was subjected to a pyrite liberation study using the AIA.

Table 2

Test Matrix for the Grinding of Liquid Nitrogen, Dry Ice, and Dry-Iced Acetone Pretreated Coal

\begin{tabular}{|llllllllll|}
\hline & \multicolumn{7}{c|}{ Grinder } & \multicolumn{1}{c|}{ Grinding Duration (min) } \\
\cline { 2 - 9 } & & Impact & 2 & 5 & 10 & 15 & 20 & 30 & 40 \\
\hline Ball Mill & & $x$ & $x$ & $x$ & $x$ & $x$ & $x$ & $x$ \\
Attrition Mill & & $x$ & $x$ & $x$ & $x$ & $x$ & $x$ & $x$ \\
Centrifugal Ball Mill & & $x$ & $x$ & $x$ & $x$ & $x$ & $x$ & $x$ \\
Impactor & $x$ & & & & & & & \\
\hline
\end{tabular}

Table 3

Test Matrix of Grinding of Pretreated Coal

\begin{tabular}{|c|c|c|c|c|c|c|c|c|c|c|}
\hline \multirow{2}{*}{$\begin{array}{c}\text { Grinding } \\
\text { Duration } \\
\text { (min) }\end{array}$} & \multicolumn{10}{|c|}{ Freezing Temperatures (K) } \\
\hline & 300 & 250 & 190 & 175 & 155 & 130 & 110 & 77 & Dry Ice & $\begin{array}{l}\text { Dry-Iced } \\
\text { Acetone }\end{array}$ \\
\hline $\begin{array}{r}2 \\
5 \\
10 \\
15 \\
20 \\
40\end{array}$ & & $\begin{array}{l}x \\
x\end{array}$ & $\begin{array}{l}x \\
x\end{array}$ & $\begin{array}{l}x \\
x\end{array}$ & $\begin{array}{l}x \\
x\end{array}$ & $\begin{array}{l}x \\
x\end{array}$ & $\begin{array}{l}x \\
x\end{array}$ & $\begin{array}{l}x \\
x \\
x \\
x \\
x \\
x\end{array}$ & $\begin{array}{l}x \\
x \\
x \\
x \\
x \\
x\end{array}$ & $\begin{array}{l}x \\
x \\
x \\
x \\
x \\
x\end{array}$ \\
\hline
\end{tabular}


Indentation fracture. Grinding of coals and/or other solids is one kind of fracturing process. To determine the ease of grinding and to evaluate its results, the kasic fracture resistance of a material must be investigated. In this study, the indentation fracture was used to determine the fracture resistance of coal as a function of the treatment temperature. In general, the indentation fracture test set-up is merely a modification of the hardness tester. In this test, a diamond pyramid indenter is used to produce a crack pattern. The loading is slowly applied to the testing material. As soon as a crack is developed, the loading is quickly released. The dimensions of the crack pattern are then observed and recorded through a high magnification microscope. The dimensions of the crack pattern and the peak loading can be used to calculate the fracture toughness of a material. The indentation test was conducted on the coal sample treated with different freezing agents and temperatures in the environmental chamber.

Microstructural investigation using scanning electron microscopy (SEM) and the automated image analyzer (AIA). The main objectives of the microstructural investigation are to: (1) identify the possible crack development along the pyrite/coal interface, (2) determine the degree of pyrite/coal liberation, and (3) perform the mineral/coal particle size distribution analysis. Basically, the SEM observation and/or microscope were applied to the samples treated at different freezing temperature conditions and to the ground coal samples. The SEM observations were performed using the facilities of the Center for Electron Microscopy at Southern Illinois University at Carbondale (SIUC). The microscope observation and AlA analysis were conducted at the Composite Materials Research Laboratory in the Department of Civil Engineering and Mechanics at SIUC. 


\section{EXPERIMENTS AND RESULTS}

The results from this project over the past two years are summarized in the following sections. However, the results related to the latest year's finding are only discussed briefly, vithout graphs and tables.

\section{Coal Selection cind Preparation}

We chose to examine the ICB-101 sample in this project. Two other coals, a Wyoming coal and a Pittsburgh Seam coal, were obtained from the DOE program. These coals were all ground to -4 mesh split and stored under nitrogen. Aliquots were ground to -20 and -100 mesh. These samples were stored under nitrogen until used. The ICB-101 sample was chosen because of its pyrite content and because of information available on size distribution of the pyrite.

\section{Freezing Temperature Pretreatments}

The main emphasis of this study was to observe possible physical changes (in particular fracture) under different low temperature conditions. The fracture study was conducted by soaking the raw coal in a freezing temperature environment. Freezing agents such as liquid nitrogen, dry ice, and dry-iced acetone have been used to introduce the freezing temperature environment. In addition, a freezing temperature chamber (which was designed to emulate the cooling chamber of the fluidized bed) was also used to pretreat coal samples at temperature levels between 77 and $300 \mathrm{~K}$. For each freezing agent used, various soaking durations $(5,10,15,20$, and $40 \mathrm{~min}$ ) were used. Only two soaking durations ( 5 and $10 \mathrm{~min}$ ) were used to pretreat coal samples using the cooling chamber. It was found that interfacial cracks between pyrite (or mineral) and coal developed as a result of the freezing temperature pretreatment. Theses interfacial cracks were lai je when compared with other types of microcracks existing in coal. It was found that the lower the 
freezing temperature the more interfacial crack development. The development of such interfacial cracks is primarily due to the different coefficients of thermal expansion in coal and in pyrite (or mirierals). The temperature change due to cooling has produced interfacial stress much higher than that a material (coal, pyrite, or minerals) would allow. It should be pointed out that the interfacial cracks imply that pyrite and minerals can be litserated rather easily. As a matter of fact, some pyrite particles were squeezed out (liberated) due to the liquid nitrogen pretreatment. The significance of the freezing ter.perature pretreatments is that the interfacial cracks can develop along the boundaries of the micro-sized (less than $10 \mu \mathrm{m}$ ) pyrite and minerals. This indicates that liberation of micro-size pyrite anci minerals may be possible without ultrafine coal grinding. A separation process may be performed immediately after the freezing temperature treatment to obtain thermally liberated micro-size pyrite and minerals particles.

Longer duration of freezing temperature soaking resulted in more extensive cracks in coal. These matrix cracks were the expansion of the interfacial cracks into coal. In general, the matrix cracks are a lot smaller than the interfacial cracks. However, the distribution of matrix cracks is very dense. It is believed that the denser the distribution of the matrix cracks, the easier it is to grind the coal. This was partially supported by the landslide fracture pattern of a liquid nitrogen pretreated coal sample. It has been shown that after being soaked in liquid nitrogen for a longer period ( $>10 \mathrm{~min}$ ) the scatter band of the particle size distribution curve became narrower.

In the dry ice and dry-iced acetone environment, microcracks also developed. However, these microcracks are predominately interfacial cracks. Very few matrix cracks were found. The interfacial cracks introduced by the dry ice and dry-iced acetone environments are much smaller in size than those introduced by the liquid 
nitrogen environment. The effectiveness of dry ice and dry-iced acetone pretreatments for the liberation of micro-size pyritic particles was low.

\section{Indentation Fracture}

The purpose of the indentation fracture testing was to determine the fracture resistance of coal under different pretreatment conditions. The Knoop hardness test was used to conduct the indentation test in this year's study. It was found that the fracture resistance of coal rapidly decreases with a decrease in the pretreatment temperature (Figure 1). There was a $50 \%$ reduction in the fracture resistance for the coal treated in a liquid nitrogen environment. Such a reduction in fracture resistance is directly related to the ease of grinding.

\section{Coal Grinding}

In this project, we have studied the effect of grinding mechanisms (different mills), grinding speeds, freezing temperature soaking time, different freezing agents (liquid nitrogen, dry ice, and dry-iced acetone), freezing temperatures, and the grinding time on the size reduction of coal. The details of the testing program have been introduced in the Experimental Procedures section.

The study of grinding using different mills was mainly to investigate the effeci. of different grinding mechanisms on the grindability of coal. An attrition mill, in general, applies shear stress to the grinding medium. On the other hand, a ball mill introduces both shear and compression. The centrifugal ball mill applies shear, compression, and impact stresses. Based on the results given in an earlier quarterly report, it was found that the centrifugal ball mill provides the best size reduction results in a similar operation condition compared to that of the ball mill and the attrition mill. The ball mill gave a better size reduction than the attrition mill. It is believed that the shearing mechanism of the attrition mill must have increased the plasticity of coal. Since the centrifugal ball mill can produce the finest particles in 


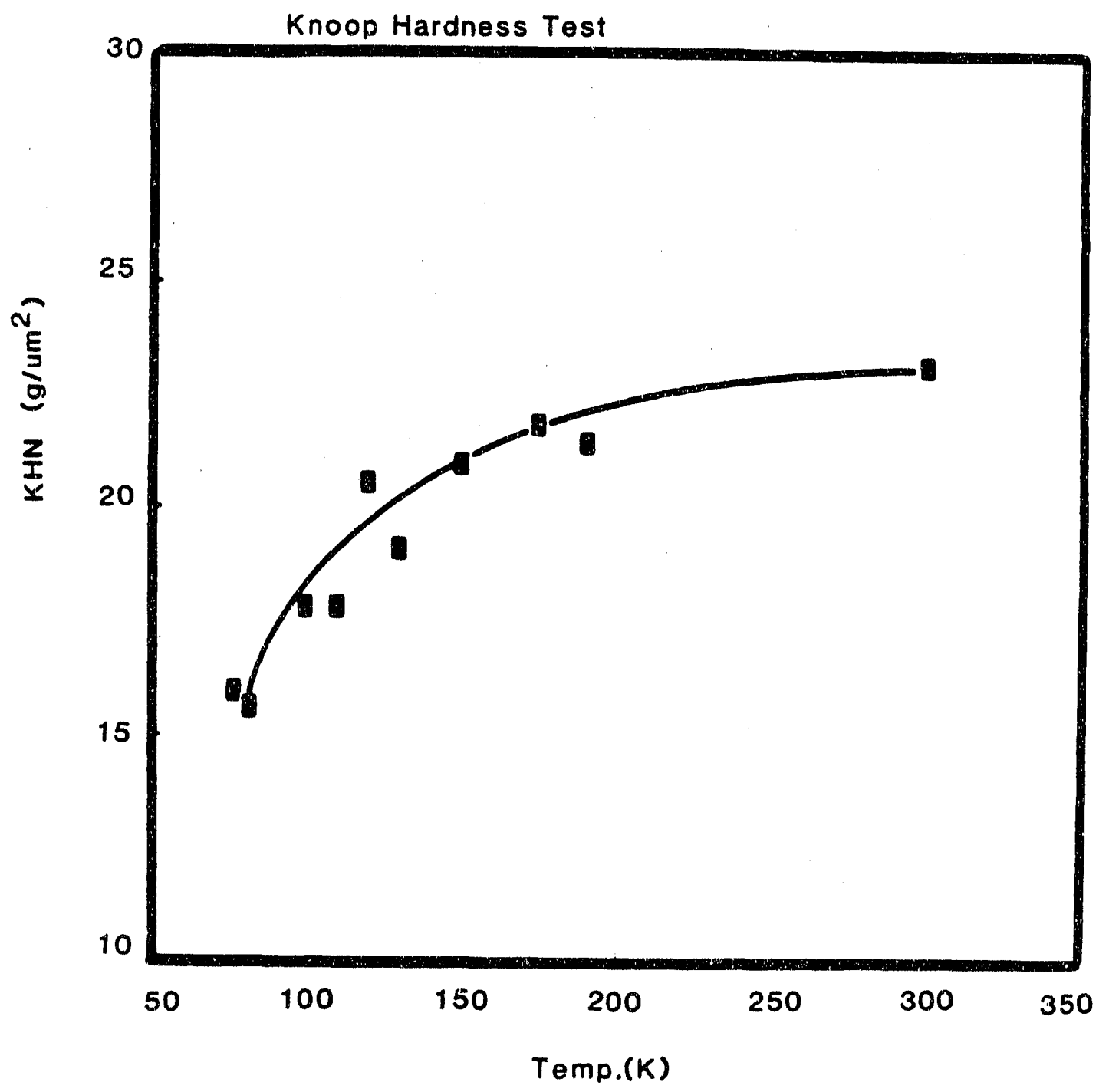

Figure 1. Fracture resistance of coal vs. the pretreatment temperature (Illinois No. 6, $10 \mathrm{~min}$ soaking). 
the shortest time among all three mills, it was used throughout this research. A 10 min grinding time was chosen to compare the grinding results obtained under different conditions. The comparison of the effect of different freezing temperature pretreatments on grindability of coal was also based on the $10 \mathrm{~min}$ grinding results.

One way of reducing (or alleviating) the deveiopment of plastic behavior is to apply load (grinding) at a faster rate. This is because most materials become less plastic-like under the high loading rate. Using this concept, coal samples were ground using the centrifugal ball mill at different rotating speeds. Indeed, it was found that additional size reduction can be obtained at a higher grinding speed. Grinding speeds between 460 and $640 \mathrm{rpm}$ were found to give the greatest size reduction for grinding using a centrifugal ball mill.

One of the main objectives of the grinding study is to determine the effect of freezing temperature pretreatment on the size reduction of coal. An assessment of the temperature range within which an effective liberation of pyrite and minerals is possible is also an objective. Three freezing agents, dry ice, dry-iced acetone, and liquid nitrogen, were used to provide the low temperature environment. In addition, an environmental chamber was built to treat coal samples at a temperature range between 300 and $77 \mathrm{~K}$. Several temperature levels (Table 1) were used to pretreat the coal samples. It was found that there was almost no improvement (over the untreated coal) of the size reduction kinematic for the dry ice treated coal. For the dry-iced acetone treated coal, a slight improvement in the size reduction was obtained. For the liquid nitrogen treated coal, a great improvement of kinematic of the particle size distribution was obtained. With liquid nitrogen pretreatment, the medium size of the ground coal is approximate $20 \mu \mathrm{m}$. It was also found that significant size reduction can be obtained as long as the treatment temperature is below 190K (Figure 2). The greatest size reduction was obtained for the samples treated 


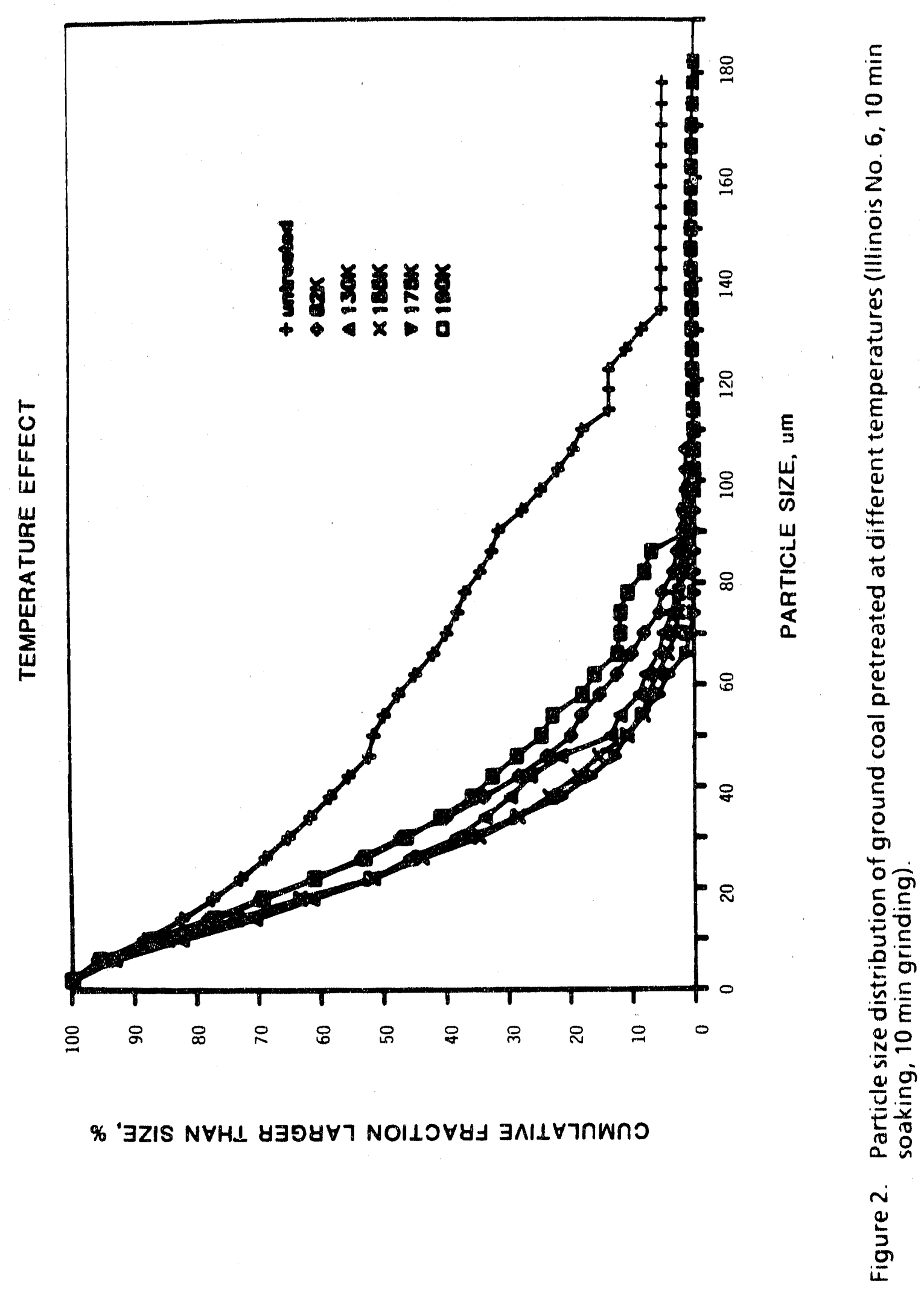


below 155K (Figure 2). This conclusion is supported by the mean particle size of the ground coal obtained through different pretreatment temperatures (Figure 3 ). It is also believed that the effectiveness of size reduction for different freezing temperature treatments has to do with the density of the matrix crack in each of the treated coals. It is believed that the denser distribution of matrix cracks will result in a greater size reduction, because it has been found that the colder the treatment temperature the more matrix cracks occur.

Since the longer duration of freezing temperature treatment wili introduce additional matrix cracks, the effect of the pretreatment time on the size reduction was investigated. A comparison of the size reduction for the coal receiving different duration of pretreatment is given in Figure 4. It was found that the size reduction increased with an increase of pretreatment duration. However, for the pretreatment duration beyond $15 \mathrm{~min}$, no significant additional size reduction was obtained.

Particle size reduction as a function of grinding time was also studied in this project. It was found that, for all the freezing temperature pretreatments, additional reduction of the medium particle size of ground coal can be obtained using a longer grinding duration (figures 5 and 6 ). However, it appears that there is a time limit beyond which grinding will not produce any additienal size reduction. It should be pointed out that the lower the pretreatment temperature the smaller the limiting value of the medium particle size.

\section{Liberation Study}

To investigate the pyrite liberation as a result of freezing temperature treatment, an AIA analysis of ground coal through a high magnification microscope was conducted. Different grinding durations and freezing temperatures resulted in different degrees of pyrite liberation. For example, figures 7 and 8 show the ground coal particles obtained in 2 and 5 min grinding, respectively, of liquid nitrogen 


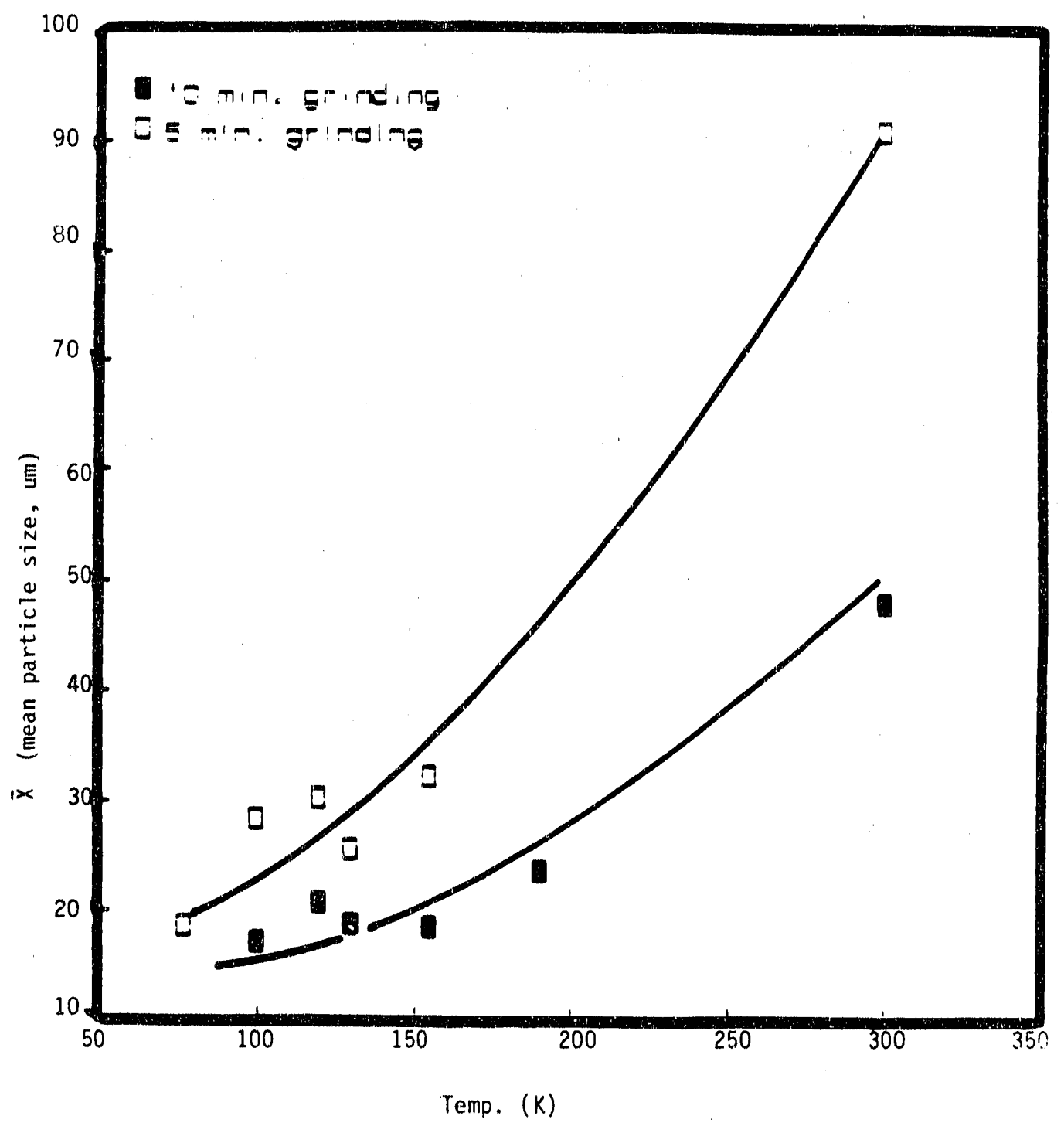

Figure 3. Mean particle size of the ground coal treated at different freezing temperatures (lllinois No. 6, 10 min soaking). 

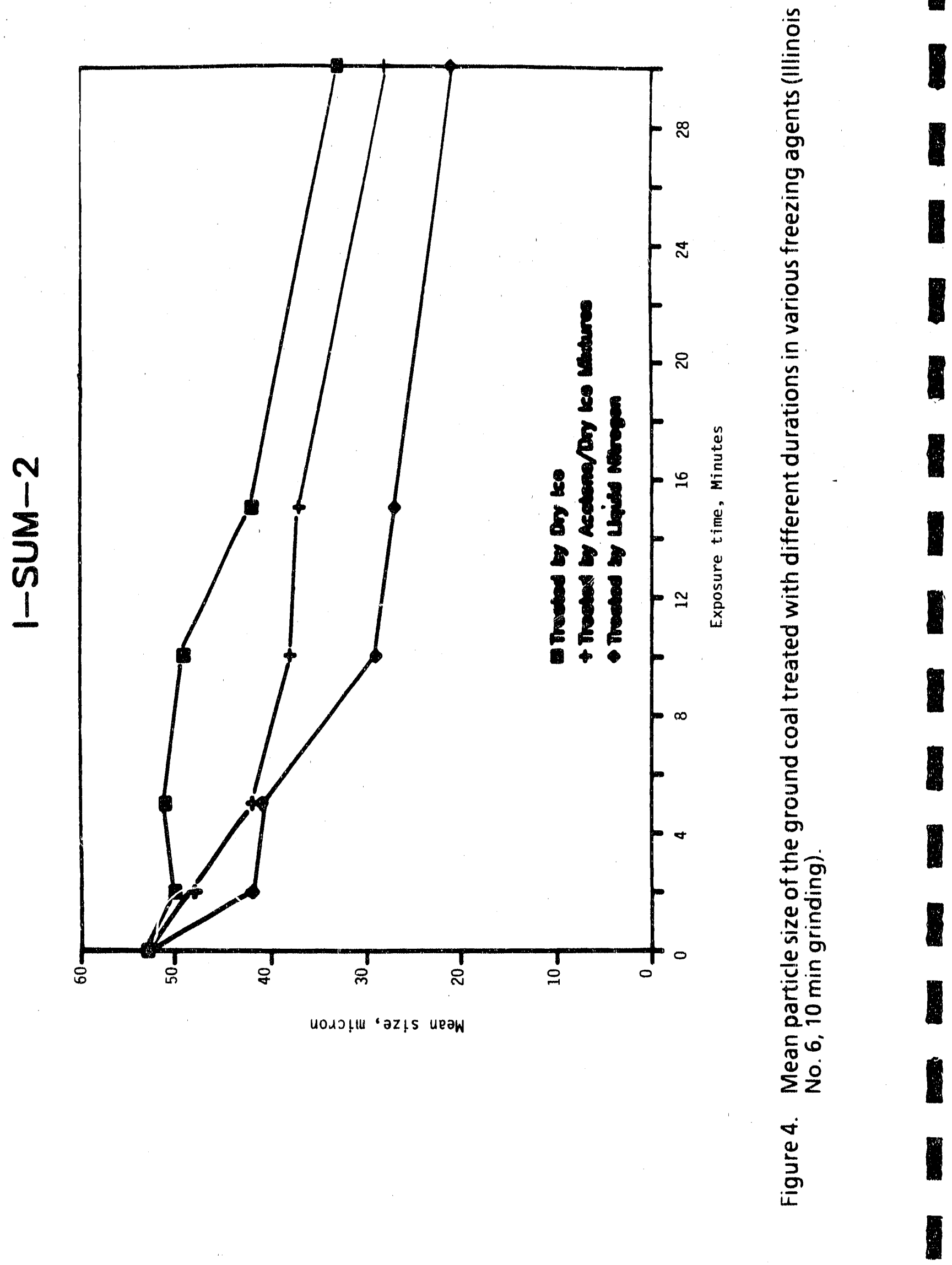


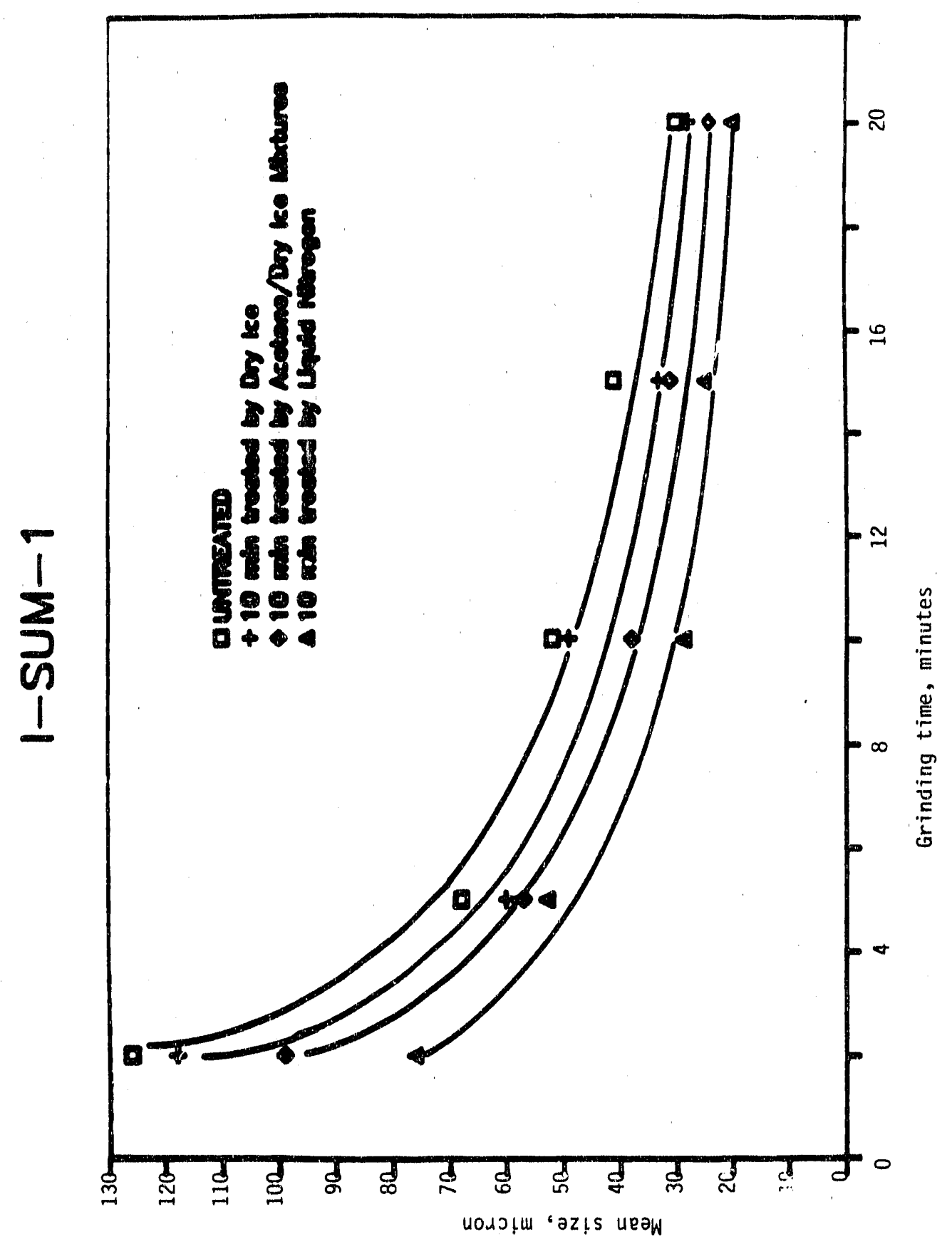

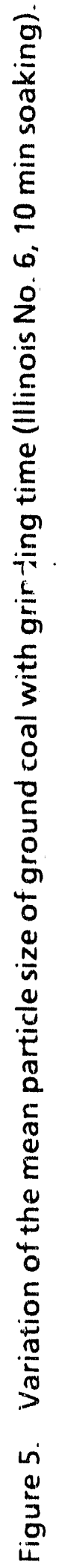




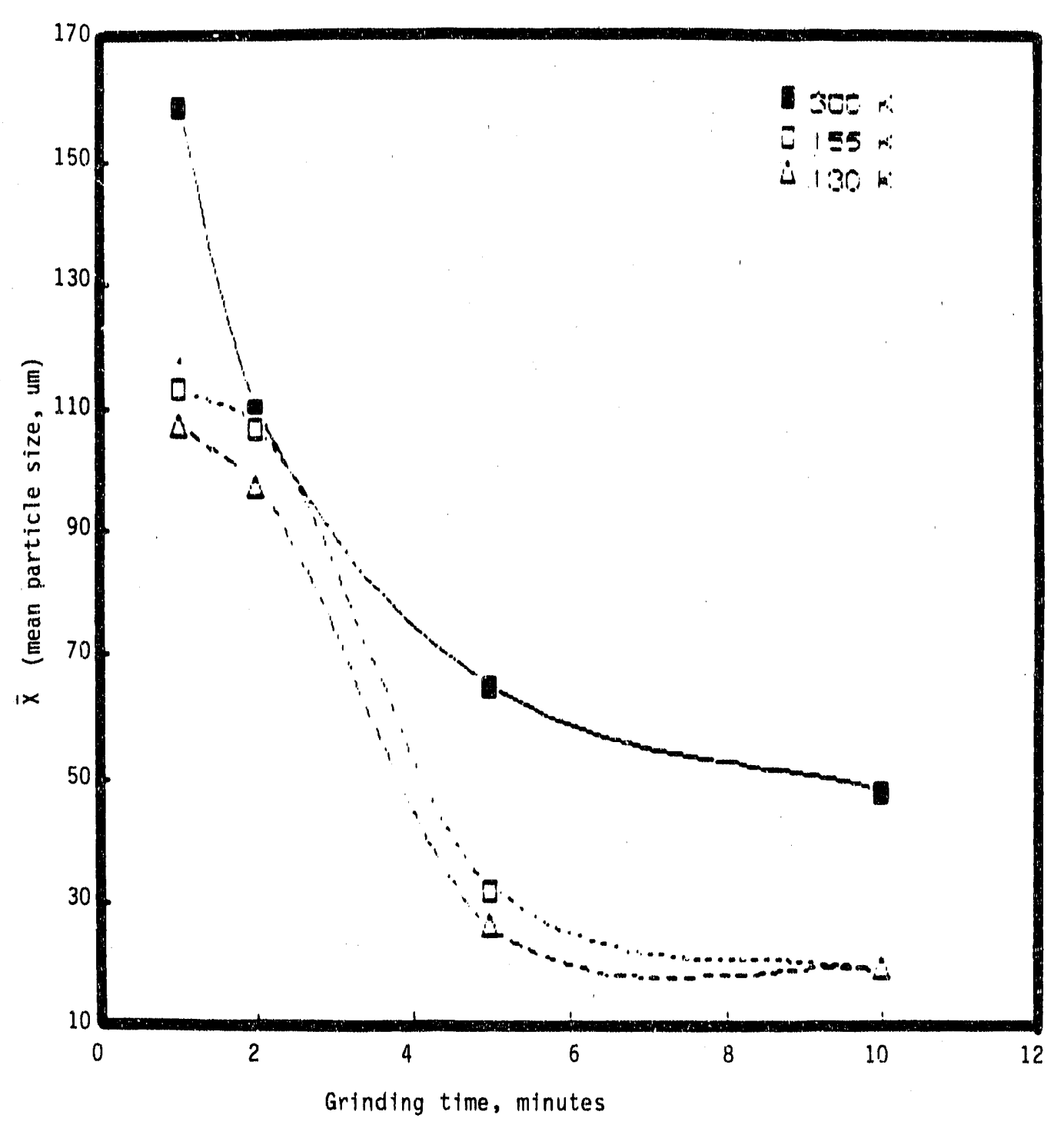

Figure 6. Variation of the mean particle size of ground coal with grinding time (IIlinois No. 6, 10 min soaking). 


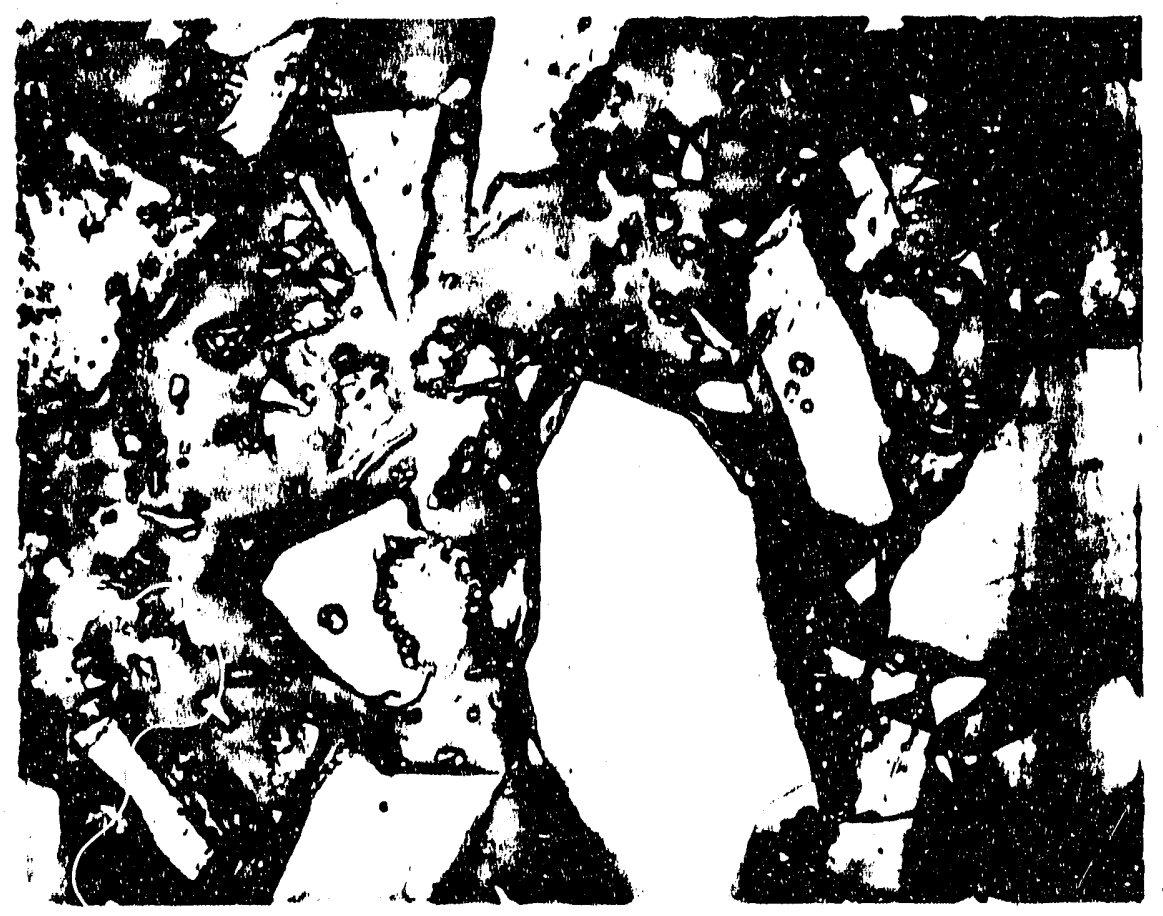

Figure 7. Ground coal powder obtained through 2 min grinding (Illinois No. 6, 10 min soaking in $L_{2}, 2$ min grinding, 1,000x).

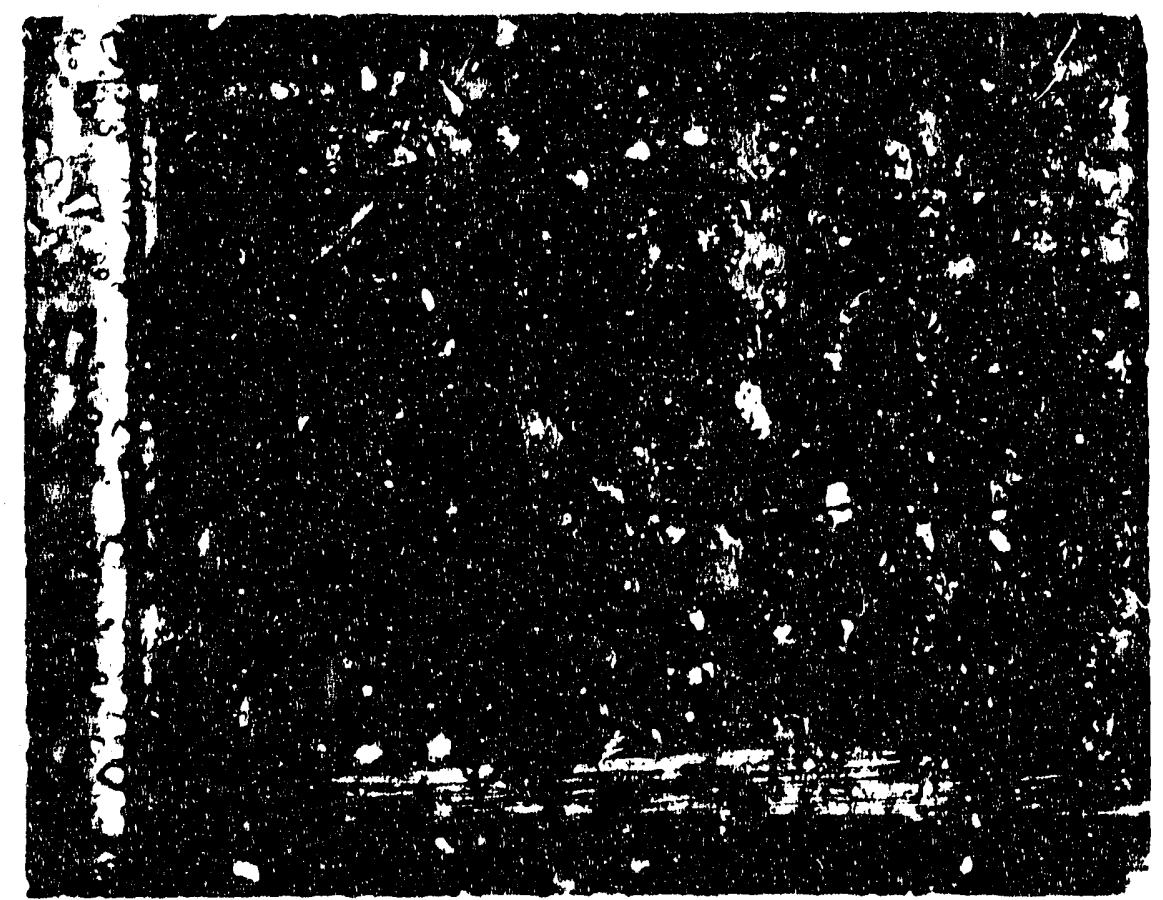

Figure 8. Ground coal powder obtained through 10 min grinding (Illinois No. 6 , 10 min soaking in $\mathrm{LN}_{2}, 10$ min grinding, $1,000 x$ ). 
pretreated coal. In these photos, the gray particles (blue/gray in Figure 7 and green/gray in Figure 8) are the coal particles while the pyrite particles are terms of gold color. The other color particles are either minerals or other macerals. It was found that there were more pyrite particles encapsulated in coal ground for $2 \mathrm{~min}$ than coal ground for $5 \mathrm{~min}$. It is obvious that the degree of pyrite liberation for the 5 min grinding is higher than that of the 2 min grinding. To give more substantiated data, extensive image analysis was conducted to calculate the amount (percentage) of pyrite liberated in each testing condition. Figures 9 and 10 show the comparison of pyrite liberation as a function of freezing temperature pretreatment time among different freezing agents (dry ice, dry-iced acetone, and liquid nitrogen) and freezing temperatures generated by the cooling chamber. The pyrite liberation of - the coal treated by dry ice and dry-iced acetone is much less significant than that of liquid nitrogen. Similarly, pyrite liberation was found to increase with a decrease in the pretreatment temperature. More than $90 \%$ of pyrite was found liberated for pretreatment temperatures lower than 130K. As much as $95 \%$ pyrite liberation was owtained in the coal treated by liquid nitrogen. Reports regarding such a high pyrite liberation have not been found in the literature. The effect of grinding time on the pyrite liberation was also studied. It was found that the longer the grinding the more pyrite liberation (Figure 11 ). However, the rate of increase in pyrite liberation decreases as grinding time increases.

It should be pointed out that the pyrite particles are, in general, much smaller than the coal particles (figures 7 and 8 ). It may be possible to define a threshold particle size (or sieve) which will result in a separation of all the liberated fine pyrite particles from relatively clean (less than $5 \%$ pyrite) larger coal particles. 


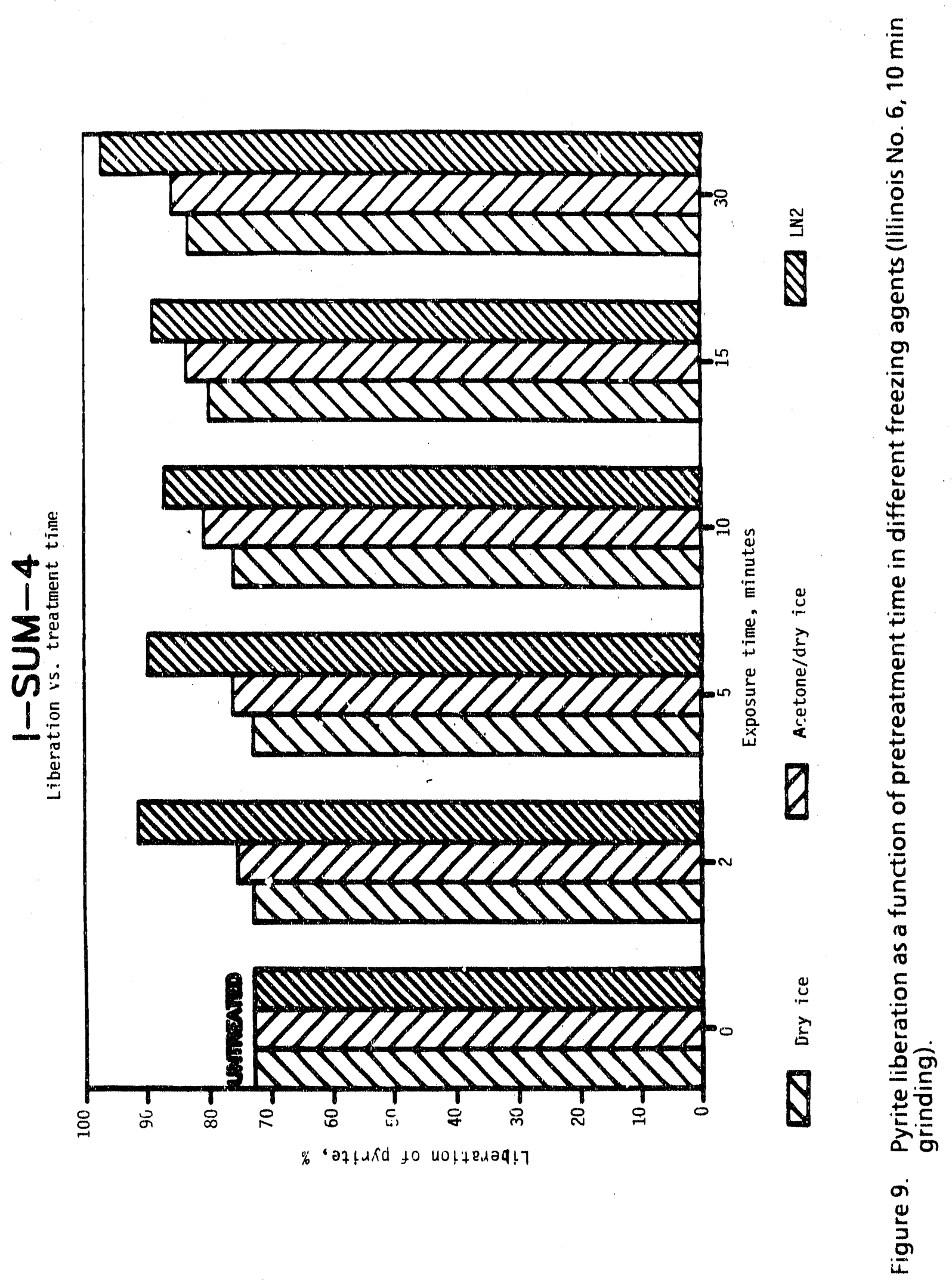



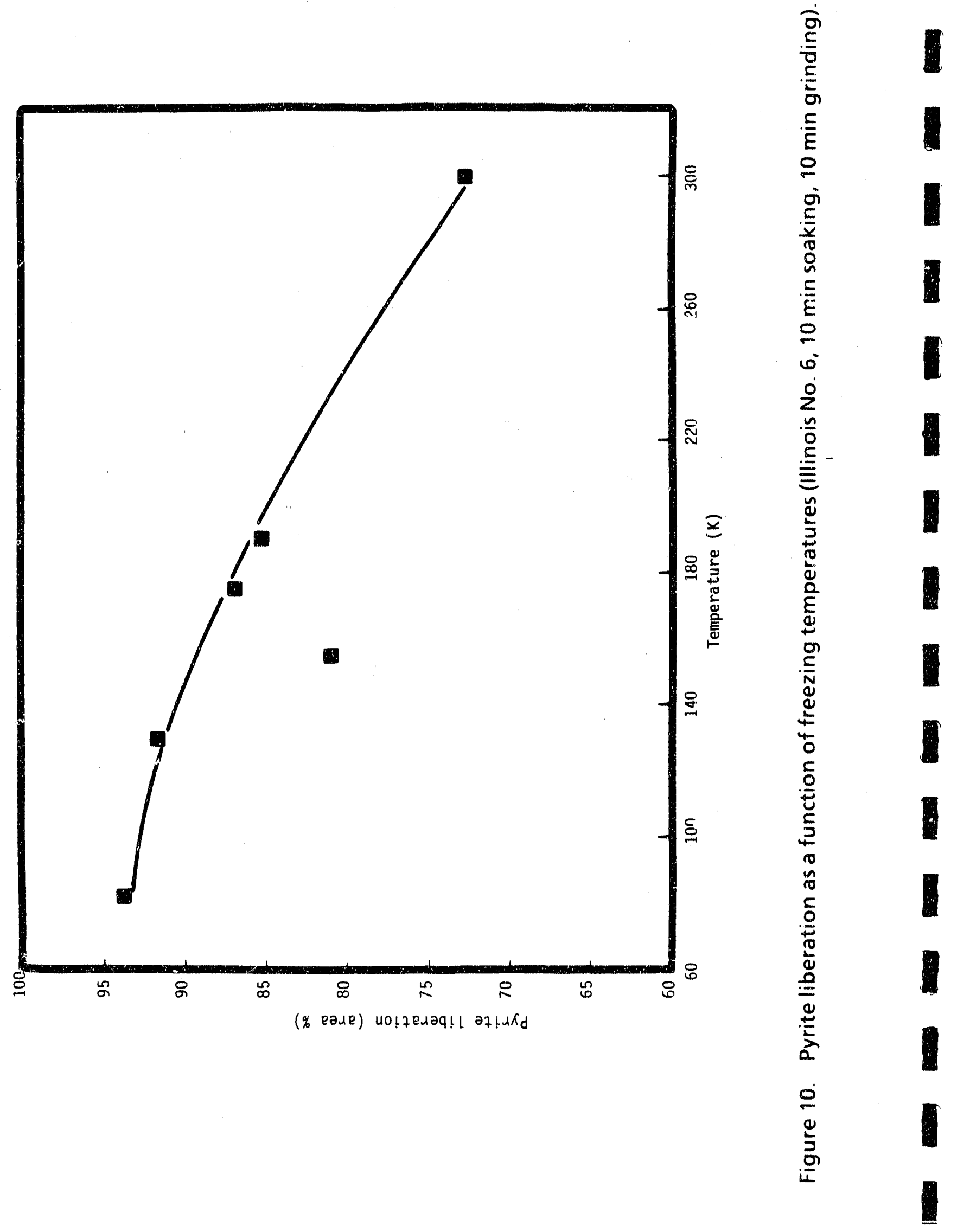


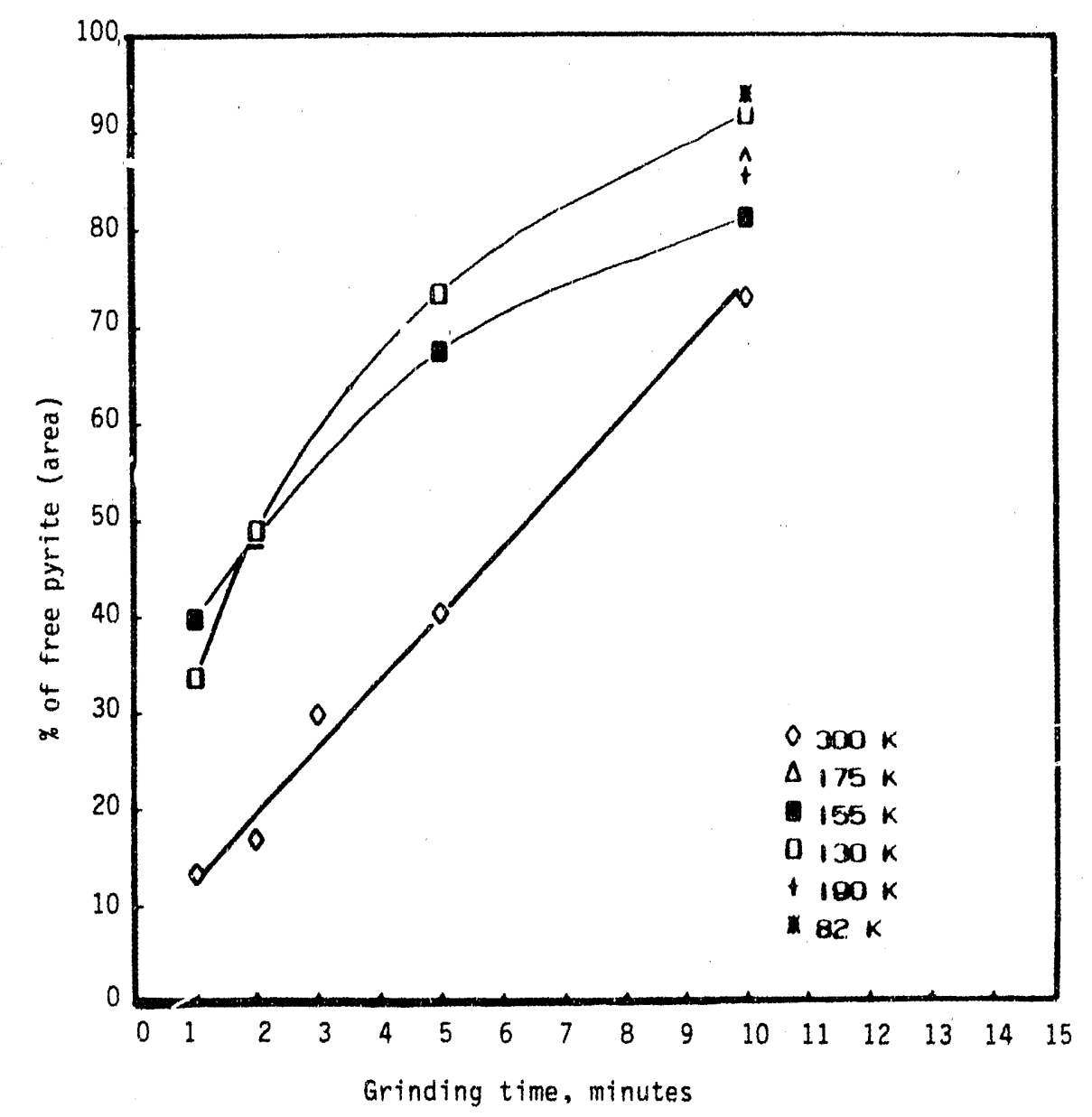

Figure 11. Pyrite liberation as a function of grinding time (IIlinois No. 6, $10 \mathrm{~min}$ soaking).

\section{Economic Analysis and Envisioned Coal Cleaning Process}

Economic evaluation and process design was started by evaluating the possibility of using liquid nitrogen as the mode of directly cooling Illinois coal. A simple thermodynamic analysis of the liquefaction energy showed that over $15 \%$ of the coal's energy would be required to produce the liquid nitrogen. When the efficiency to generate electricity was added the energy cost was greater than $35 \%$ of the coal's energy. Clearly, using only liquid nitrogen is inefficient. Not enough energy could be saved in the cooling cycle to consider liquid nitrogen with gas pre-cooling. Furthermore, direct contact of pure nitrogen with coal would result in contamination of 
the gas/liquid nitrogen with oxygen trapped on the coal surface, as it would enter the process. This means that the nitrogen would have to be dehumidified (not a difficult problem) and purified (removal of the oxygen from the nitrogen would be costly from thermodynamic and economic prospects). Direct contact is necessary to maintain appropriately large heat transfer between the coal and the refrigerant. It is inappropriate to present all the calculations here, but a solid coal to solid exchanger wall heat transfer would result in a minimal transfer coefficient and a prohibitive heat transfer area.

Next air was considered as the refrigerant contact. This seems to be a better choice but the temperature range needs to be restricted so that oxygen will not concentrate in a portion of the system. In other words the temperature of operation must be kept above $90 \mathrm{~K}$ (the boiling point of oxygen at $1 \mathrm{~atm}$ ). Having condensed oxygen in the system would be dangerous and, as it turns out, unnecessary. In addition, the higher this lower temperature the more efficient the refrigeration can be made.

If we assume that no refrigeration recovery is to be used in the process, approximately $90 \mathrm{kcal} / \mathrm{kg}$ of coal is required to lower the temperature of the coal. This estimated cooling requirement is based on the following: (1) the entering coal must be cooled from an average inlet temperature of $310 \mathrm{~K}$ to $130 \mathrm{~K}$, and (2) the average heat capacity of the coal is $0.5 \mathrm{~kg}$ over this temperature range (this value was obtained from V. Malhotra, Department of Physics at SIUC, and is actual DSC data that was reported to the Center for Research on Sulfur in Coal). A critical factor in the design is the temperature to which coal must be cooled in order to obtain the most appropriate brittleness to give selective liberation of pyrite and other coal minerals. This can be observed by considering the envisioned process in more detail.

Presently, a fluidized bed type coal cleaning system is under consideration. This system design was based on the concept that the freezing temperature environment 
will enhance the grindability of coal, and that, after treatment with very low temperature, only very mild grinding is needed for liberation of pyrite and other minerals. In the cleaning system, components such as the grinder, compressor, cyclone, feed hopper, and heat exchangers are necessary. A piping system must be considered for circulation of cold and warm gases. In addition, several separation stations may be necessary for different stages of selectivf: separation.

A grinder will be placed in the fluidized bed to grind the embrittled coal and entrained partially ground coal in a lift tube where the coal will be impacted against a diverter plate. The coal will be shattered, selectively liberating the minerals as fines and producing intermediate size coal particles. The fines will be ejected from the bed and removed in a cyclone. Coal may need to be recovered from these trapped fines by an advanced cleaning method but the volume will be a fraction of the original feed coal. Thus a great deal of cost savings will be obtained from reducing the amount of coal to be taken to advanced cleaning. We estimate that $10 \%$ organic material may accompany the $10 \%$ mineral component. Thus only $10 \%$ of the coal would see advanced cleaning. Therefore advanced coal cleaning cost should be reduced by a factor of 5 or 10 on a per ton of coal feed basis. To clean the entire ton might cost $\$ 20$ to $\$ 40$ per ton; thus advanced coal cleaning cost might be reduced to $\$ 2$ to $\$ 8$ per ton of feed coal.

Two components, a freezing temperature treatment chamber (Figure 12) and an impact grinder (Figure 13) that emulate the parts in the fluidized bed system were designed and built. The cooling chamber utilized different mixtures of nitrogen and liquid nitrogen gases to introduce different freezing temperatures. This cooling chamber has been used to chill coal samples at various temperature levels between 300 and $77 \mathrm{~K}$. The impact grinder utilizes the high pressure gradient between two ends of a pipe to drive pretreated coal particles to smash against a very rigid steel plate. It was found that -325 mesh particles were obtained through the 


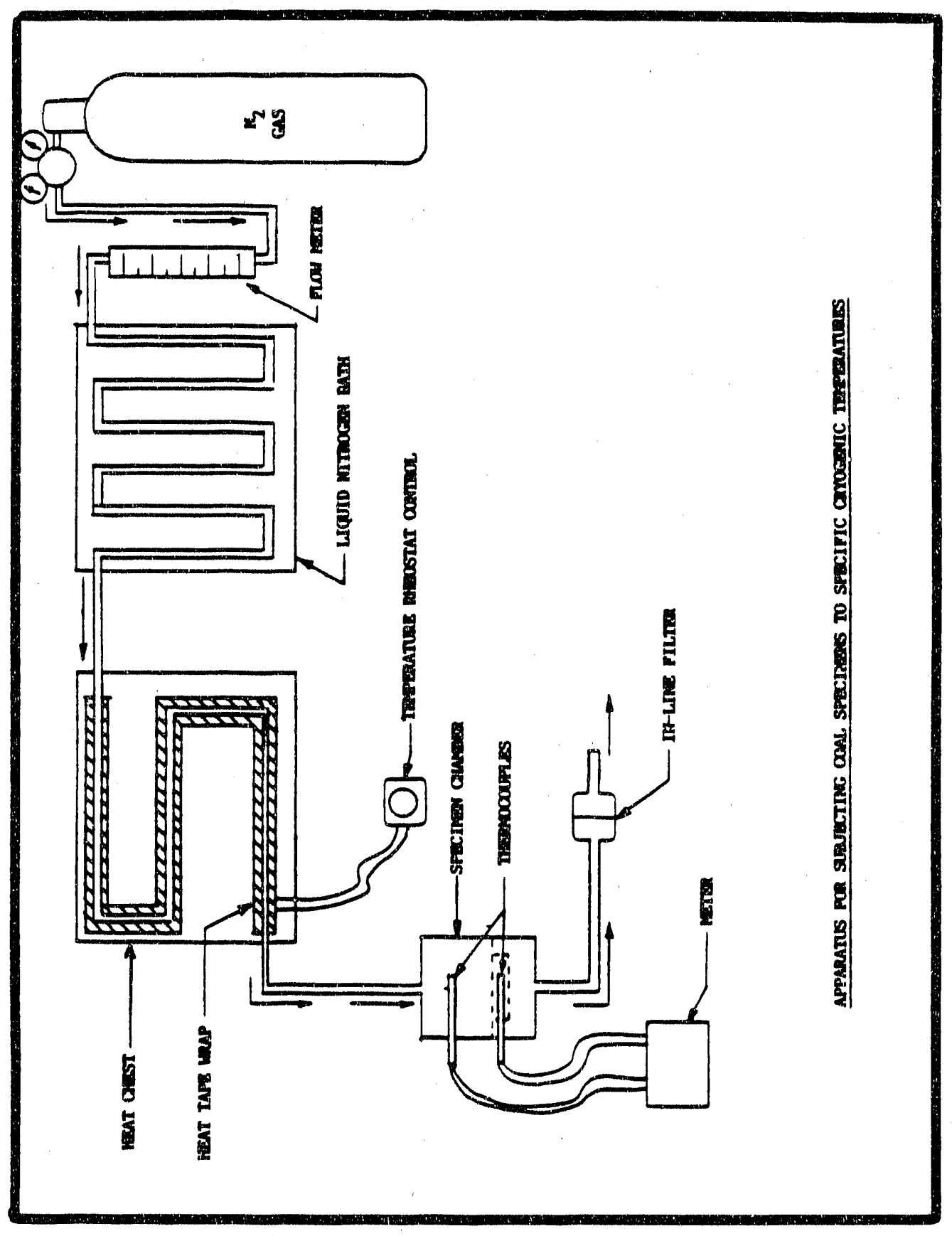

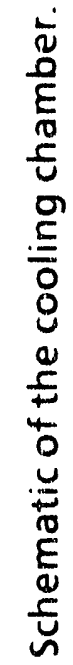

ำ 


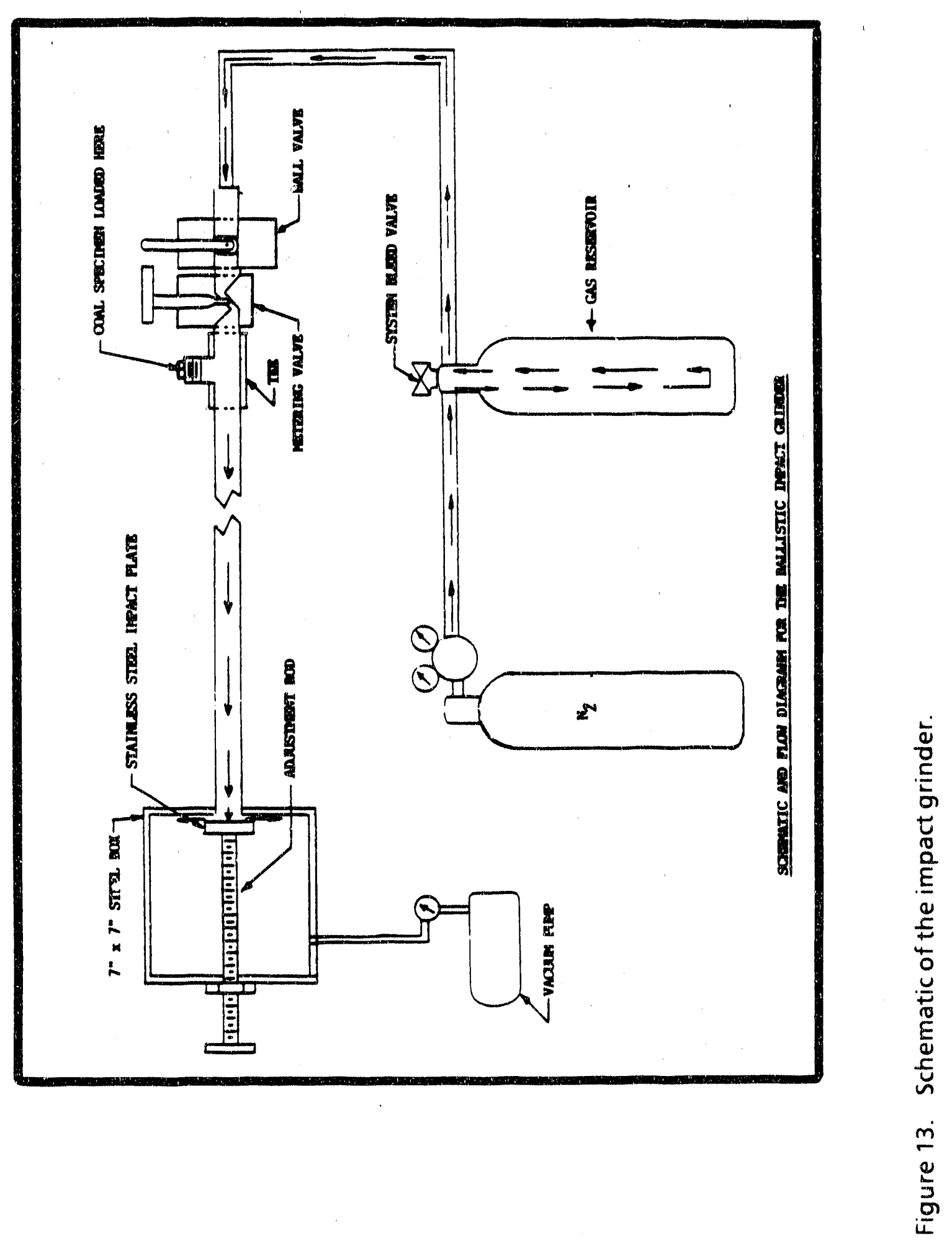


impact of a liquid nitrogen pretreated coal sample. It appears that a large portion of the -325 mesh ground coal particles is pyrite or minerals. Very few pyrite particles were found in the +325 portion of this ground coal sample.

\section{CONCLUSIONS}

Based on the results obtained in the past two years, the following is a summary of the conclusions of this project.

The freezing temperature can introduce interfacial and matrix cracks which enhance the grindability of coal.

The particle size distribution of ground coal can be controlled by the time and temperature of the freezing temperature pretreatment, the grinding time and speed, and the grinding mill. A selective grinding process must consider these parameters.

More pyrite liberation can be obtained through the freezing temperature pretreatment process. As much as $95 \%$ pyrite liberation was obtained for the liquid nitrogen pretreated coal.

Application of freezing temperature pretreatment to enhance pyrite liberation in coal is a feasible process. 


\section{REFERENCES}

Austin, L. G., R. R. Klimpel, and P. T. Luckie. 1984. In Process engineering of size reduction: Ball milling, pp. 21-37. Society of Mining Engineers, AIMMPE.

Bose, D. G. 1983. Ultrafine grinding equipment, types, capacities, and choice. In Ultrafine-grinding and separation of industrial minerals, ed. G. G. Malgham, pp. 23-28. Society of Mining Engineers, AIMMPE.

Grady, W. C. 1977. Microscopic varieties of pyrite in West Virginia coal. Transactions Of AIMMPE 262:268-274

Hamblem, D. G., P. R. Solomon, and R. H. Hobbs. 1980. Physical and chemical characteristics of coal. EPA-600/7-80-106. Washington, D.C.: U.S. Environmental Protection Agency.

Hartwig, G. 1978. Low-temperature properties of epoxy resins and composites. In Advances in cryogenic engineering, vol. 24, eds. K. D. Timmerhaus, R. P. Reed, and A. F. Clark, pp. 17-36. Plenum Press.

Hsieh, S. 1976. Effects of bulk-components on grindability of coals. Ph.D. dissertation, The Pennsylvania State University.

Klimpel, R. R. 1987. Fine coal grinding. In Fine coal processing, eds. S. K. Mishra and R. R. Klimpel, pp. 19-56. Noyes Publications.

Kowalczewski, J. J., P. C. Bandopadhayay, R. J. Downie, and N. R. Read. 1984. Ultrafine grinding of Australian brown coal for use in diesel engines. In Coal slurry combustion and technology, vol. I. Pittsburgh, Pennsylvania: U.S. Department of Energy.

McCarthey, J. T., H. J. O'Donnell, and S. Ergun. 1969. Pyrite size distribution and coalpyrite particle association in steam coals. Report of Investigation No. 7231. U.S. Bureau of Mines.

Parish, B. M. 1967. The effect of rank and particle size on the plastic behavior of coal. British Journal of Applied Physics 18:233-240.

Richardson, D., and H. L. Love, II. 1979. Pyrite liberation in coal key to sulfur reduction during beneficiation. In Coal Preparation and Utilization Symposium, Preprints, p. 10. McGraw-Hill, Inc.

Smith, F. J., and J. R. Odekirk. 1985. Ultrafine coal characterization. Second quarterly report, DOE Contract DE-AC22-84PC72007. Pittsburgh, Pennsylvania: U.S. Department of Energy.

U.S. Department of Energy, Pittsburgh Energy Technology Center. 1987. Coal surface control for advanced physical fine coal cleaning technologies. Request for Proposal Number DE-RP22-88PC79871. 
Yen, S. C., and E. J. Hippo. 1989a. Comminution employing liquid nitrogen pretreatments. Coal Technology Laboratory first quarterly report. Carbondale: Southern Illinois University at Carbondale, Coal Research Center.

Yen, S. C., and E. J. Hippo. 1989b. Comminution employing liquid nitrogen pretreatments. Coal Technology Laboratory second quarterly report. Carbondale: Southern illinois University at Carbondale, Coal Research Center. 

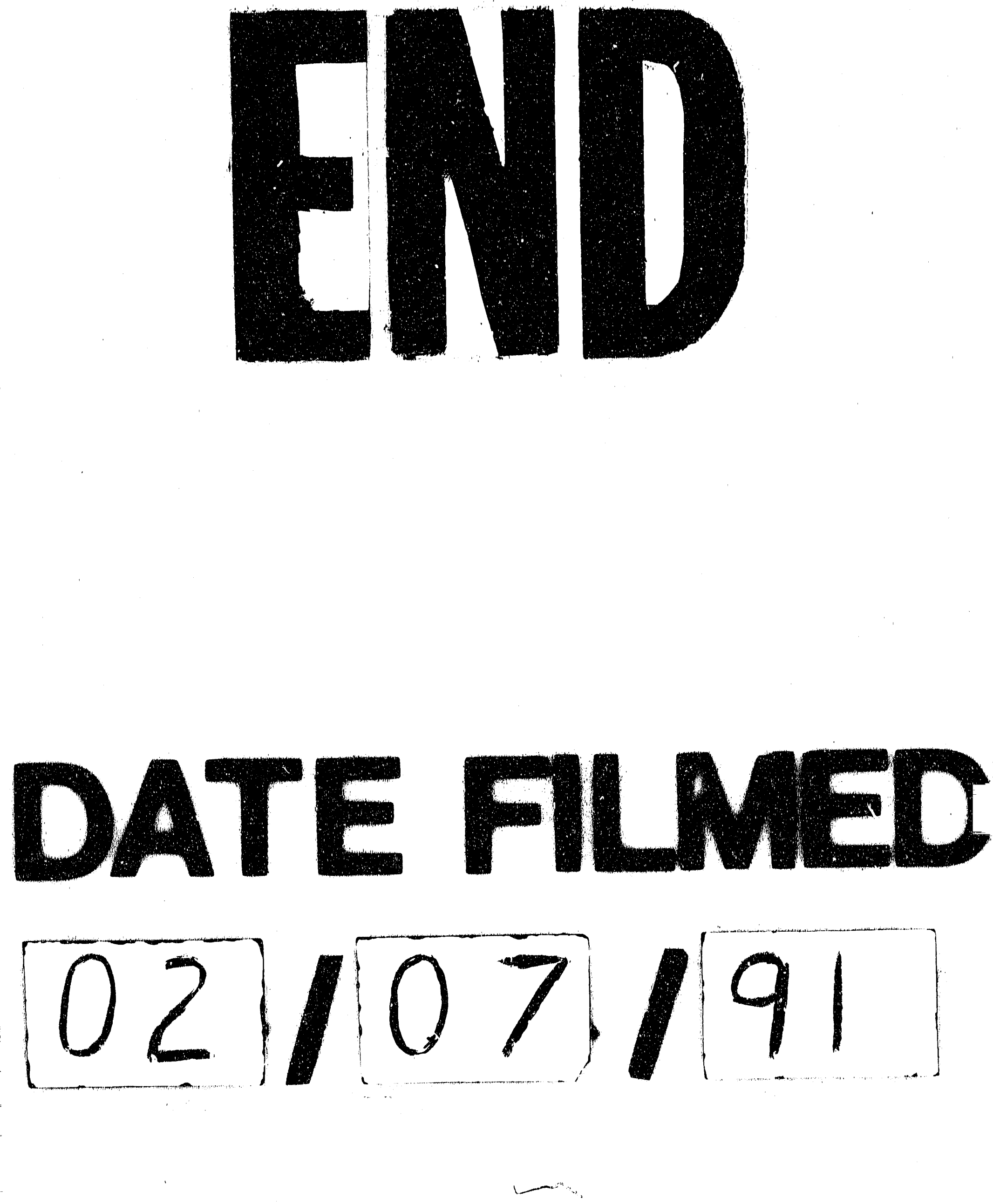
